\title{
The clinical and economic burden of community- onset complicated skin and skin structure infections in Korea
}

\author{
Yong Kyun Cho ${ }^{1}$, Heung Jeong Woo ${ }^{2}$, Shin Woo Kim³ ${ }^{3}$ In-Gyu Bae ${ }^{4}$, Young Goo Song 5 , Hee Jin Cheong ${ }^{6}$, \\ Hyuck Lee ${ }^{7}$, Sang Hoon $\mathrm{Han}^{5}$, Hee Jung Choi ${ }^{8}$, Chisook Moon ${ }^{9}$, Seong Yeol Ryu ${ }^{10}$, Jian Hur ${ }^{11}$, \\ Ja Cob Lee ${ }^{2}, \mathrm{Yu} \mathrm{Mi} \mathrm{Jo}{ }^{1}$, and Young Joo Kim ${ }^{12}$
}

\begin{abstract}
Department of Internal Medicine, ${ }^{1}$ Gachon University College of Medicine, Incheon; ${ }^{2} \mathrm{Hallym}$ University College of Medicine, Chuncheon; ${ }^{3}$ School of Medicine, Kyungpook National University, Daegu; ${ }^{4}$ Gyeongsang National University College of Medicine, Jinju; ${ }^{5}$ Yonsei University College of Medicine, Seoul; ${ }^{6}$ Korea University College of Medicine, Seoul; ${ }^{7}$ Dong-A University College of Medicine, Busan; ${ }^{8}$ Ewha Womans University College of Medicine, Seoul; ${ }^{9}$ Inje University College of Medicine, Busan; ${ }^{10}$ Keimyung University College of Medicine, Daegu; ${ }^{11}$ Yeungnam University College of Medicine, Daegu; ${ }^{12}$ Pfizer Pharmaceuticals Korea Ltd., Seoul, Korea
\end{abstract}

Received: October 16, 2018 Revised : February 28, 2019 Accepted: March 13, 2019

\section{Correspondence to Yong Kyun Cho, M.D. Department of Internal Medicine, Gachon University Gil Medical Center, 21 Namdong- daero 774beon-gil, Namdong-gu, Incheon 21565 , Korea \\ Tel: $+82-32-460-8447$ \\ Fax: $+82-32-460-8448$ \\ E-mail: karmacho@gmail.com}

Background/Aims: To investigate epidemiologic characteristics, clinical and economic burdens, and factors associated with mortality in complicated skin and skin structure infection (cSSSI) patients in Korea.

Methods: A retrospective, observational, nationwide study was conducted between April to July 2012 at 14 tertiary-hospitals in Korea. Eligible patients were hospitalized adults with community acquired cSSSI, who underwent surgical intervention and completed treatment between November 2009 and October 2011. Data on demography, clinical characteristics, outcomes and medical resource utilization were collected through medical record review. Direct medical costs were calculated by multiplying quantities of resources utilized by each unit price in Korea.

Results: Of 473 patients enrolled, 449 patients (except 24 patients with no record on surgical intervention) were eligible for analysis. Microbiological testing was performed on $66.1 \%$ of patients and $8.2 \%$ had multiple pathogens. Among culture confirmed pathogens ( $\mathrm{n}=297$ patients, 340 episodes), $76.2 \%$ were gram-positive (Staphylococcus aureus; $41.2 \%$ ) and $23.8 \%$ were gram-negative. The median duration of hospital stay was 16 days. Among treated patients, 3.3\% experienced recurrence and $4.2 \%$ died in-hospital. The mean direct medical costs amounted to $\$ 4,195 /$ person, with the greatest expenses for hospitalization and antibiotics. The in-hospital mortality and total medical costs were higher in combined antibiotics therapy than monotherapy $(p<0.05)$. Charlson's comorbidity index $\geq 3$, standardized early warning scoring $\geq 4$, sub-fascia infections and combined initial therapy, were all found to be associated with higher mortality.

Conclusions: Korean patients with community-onset cSSSI suffer from considerable clinical and economic burden. Efforts should be made to reduce this burden through appropriate initial treatment.

Keywords: Complicated skin and skin structure infection; Economic; Clinical burden; Initial antibiotics 


\section{INTRODUCTION}

Skin and skin structure infection (SSSI) is an infection of the skin and associated tissues, and thus, the equivalent term of skin and soft tissue infections is identically used. According to United States data, hospital admissions for adult patients due to SSSI are increasing [1]. The term 'complicated' SSSI (cSSSI) is often used when the infection requires surgical intervention and/or the infectious process involves (suspected or confirmed) deeper soft tissue; however, there is no universally accepted criteria for defining cSSSIs [2-4]. Complicated SSSIs usually include secondary infections of diseased, acute wound infections (traumatic, bite-related), surgical site infections (deep, organ-space), chronic wound infections (diabetic foot, venous stasis ulcers, pressure sores) and perianal cellulitis [5].

The etiology of CSSSIs is usually polymicrobial in nature. Most commonly implicated pathogens are gram positive cocci, and particularly Staphylococcus aureus. Gram negative bacilli and anaerobic bacteria can be encountered in more complicated cases of cSSSI [4-6]. These etiological differences among CSSSIs can depend on the type of infection, as well as if the infection is community-acquired or nosocomial [7].

Complicated SSSIs have a high disease burden and are sometimes difficult to diagnose. Hence, cSSSIs may progress to severe necrotizing fasciitis entailing subcutaneous connective tissues and stretch deep into other soft tissues $[8,9]$. These infections may be inexorably progressive despite treatment even when limb-or life-threatening [5]. An estimated $3 \%$ of cSSSI patients face death and the mortality rate increases sharply to $29 \%$ when it progresses to necrotizing fasciitis $[9,10]$. The high economic burden of treatment further exacerbates the problem; treatment costs are estimated to be as high as United States dollar (USD) 48,033 per person, which increases if the patient has additional procedures, ICU admission, or any other treatments [11]. It follows that prompt diagnosis and initiation of effective antibiotic therapy is crucial in the management of cSSSI patients.

Only a few studies have assessed the burden of cSSSI infections and factors incurring additional burden on patients with cSSSI in Korea. This study was conducted to provide the following information regarding community-onset cSSSIs in Korea: (1) epidemiologic in- formation, (2) clinical outcomes of treatment, (3) direct medical cost associated with treatment, and (4) factors associated with mortality in cSSSI.

\section{METHODS}

This retrospective, observational, nationwide study was conducted at 14 tertiary hospitals in Korea between April 2012 and July 2012. The study was approved by the Institutional Review Boards (IRBs) of all participating hospitals. All procedures have, therefore, been performed in accordance with the ethical standards of the institutional and/or national research committees and with the 1964 Declaration of Helsinki and its later amendments or comparable ethics standards. The IRB approval number of the Gachon University College of Medicine, which is responsible for this study, is GIRBA-2655-2012. Written informed consents was waived a retrospective nature of our study by the board. The IRB numbers for the other participating hospitals are as follows: KUGH11243-001, 2011-280, 2011-12-123, 4-2011-0735; ECT 12-O2A-29, 3-2011-0276; PCR-11-202, 11-317 12.16; KNUH 2011-12-019, 11-91, 11-199, 11-186, 2012-01-006.

\section{Patients}

Patients were considered eligible for enrollment if they met all of the following criteria: (1) aged $\geq 18$ years; (2) required surgical interventions and were admitted to hospitals with the diagnosis of community-onset cSSSI according to the following international classification of disease (ICD)-10 codes; major abscess (Lo2.0 to L02.9), necrotizing cellulitis (L03.0 to L03.9), cellulitis merged with deep myositis (M6o.o, M6o.8, M6o.9), pyomyositis (M60.0, M60.8, M60.9), necrotizing fasciitis (M72.6) and traumatic infection (T79.3); (3) culture-confirmed infection within 48 hours of hospitalization or underwent treatment for culture-confirmed infection from the surgical specimen.

Patients who met at least one of following criteria were excluded: (1) experienced an uncomplicated skin and skin structure infection; (2) had hospital acquired cSSSI; (3) recovered after treatment of more than 2 days in another hospital; (4) transferred to another hospital during treatment or was discharged against medical advice; (5) presented with osteomyelitis close to the infect- 
ed area or deep site tissue infection other than skin and skin structure infection (e.g., deep neck infection, psoas abscess); (6) received plasmapheresis or hemoperfusion; (7) had concurrent infection of non-removable prosthetic materials (e.g., permanent cardiac pacemaker battery packs or joint replacement prostheses); (8) suffered from neutropenia (absolute neutrophil count $<500$ cells $/ \mathrm{mm}^{3}$ ) or human immunodeficiency virus of $\mathrm{CD}_{4}$ count $<100$ cells $/ \mathrm{mm}^{3}$ within the most recent 6 months; (9) received more than two immunosuppressants or received prednisolone dosage of $40 \mathrm{mg}$ daily (or equivalent) for more than once a week within the most recent 3 months; (10) received chemotherapy or radiotherapy within the most recent 3 months; (11) was participating in some other clinical research; or (12) was deemed unsuitable for the study as judged by the researcher (e.g., tuberculosis or nontuberculous mycobacterial infection, fungal infection, Vibrio vulnificus sepsis patient, etc.).

The 473 patients with cSSSI who completed treatment between November 2009 and October 2011 were enrolled and the number of enrolled patients in each hospital was restricted to a maximum of 40 patients. Of the 473 patients, 24 patients who did not record the surgical intervention in their case report form were excluded. The remaining 449 patients who met the inclusion criteria were analyzed in our study. Most patients' medical charts were reviewed for cSSSI treatment throughout their first and last hospital visits.

\section{Data collection \& quantification of medical resource utilization}

The following data were collected through the medical chart review:(1) demography data including age and gender; (2) clinical characteristics including disease severity (standardized early warning scoring [SEWS]), comorbidities (Charlson's comorbidity index, CCI), and data on infections and its treatment; (3) clinical outcomes data including length of hospital stay, admission to intensive care unit (ICU), recurrence within 3 months of the treatment completion and in-hospital mortality (defined as death related and/or non-related with cSSSI).

All medical resource utilizations during the entire cSSSI treatment period were collected by retrospective review of patient medical records. The cSSSI treatment period was defined as the period between the first and last hospital visit. The direct medical costs were calcu- lated by multiplying quantities of resources utilized by the unit price of each resource. The unit price of each resource was obtained from the Health Insurance Price List (Korea HIRA 2010), and the medication cost was from the weighted average cost (2010). Categories of costs included hospitalization (outpatient visit, emergency department visit, intensive care unit admission and general department admission), antibiotics, other medications (other than antibiotics), laboratory tests (blood and urine tests), imaging tests (X-ray, computed tomography, magnetic resonance imaging, sonography, etc.), diagnostic tests (culture, gram stain through blood culture, wound or drainage, etc. for etiological diagnosis), and surgeries and procedures (e.g., incision \& drainage, etc.). All costs were presented in USD (\$) using the exchange rate of $1,100 \mathrm{KRW}=\$ 1$; this exchange rate approximately corresponds to the value of the average exchange rate of USD during the past 10 years.

\section{Statistical analysis}

Descriptive analysis was performed to assess demographic and clinical characteristics, distribution of pathogens, and clinical and economic burden of disease. Continuous variables were shown as the mean with standard deviation $(\mathrm{SD})$, or median with interquartile range (IQR). Median with IQR was used to represent values when the data were not following a normal distribution. The cost was log-transformed since it did not follow a normal distribution. Frequency and proportions were calculated for categorical variables. Chi-square test was used to evaluate differences in CCI, SEWS, depth of infection and clinical burden (except hospital length of stay) between initial therapy types (monotherapy and combined therapy). Hospital length of stay between initial therapy types was compared with the Mann-Whitney test.

Logistic regression was performed to identify risk factors that were associated with the mortality of community-onset cSSSI patients. The candidate variables, which were anticipated to be associated with mortality, were selected based on the literature and clinical experiences, and included in the model. Then, the variables were selected by the backward elimination method.

A two-sided $p$ value of 0.05 was considered statistically significant. All statistical analyses were performed using SPSS statistical program version 20.o (IBM Co., Armonk, NY, USA). 
Table 1. Characteristics of study patients and clinical burden of community-onset complicated skin and skin structure infections $(n=449)$

\begin{tabular}{|c|c|}
\hline Characteristic & Value \\
\hline Age, yr & $54.6 \pm 16.8$ \\
\hline Male sex & $290(64.6)$ \\
\hline \multicolumn{2}{|c|}{ Standardized early warning score at admission } \\
\hline $0-1$ & $368(82.0)$ \\
\hline $2-3$ & $47(10.5)$ \\
\hline $4-6$ & $27(6.0)$ \\
\hline$\geq 7$ & $7(1.5)$ \\
\hline \multicolumn{2}{|l|}{ Charlson comorbidity index } \\
\hline o & $247(55.0)$ \\
\hline $1-2$ & $147(32.7)$ \\
\hline $3-4$ & $37(8.3)$ \\
\hline$\geq 5$ & $18(4.0)$ \\
\hline \multicolumn{2}{|l|}{ Infections } \\
\hline \multicolumn{2}{|l|}{ Type of infection ${ }^{a}$} \\
\hline Major abscess & $233(51.9)$ \\
\hline Necrotizing cellulitis & $144(31.1)$ \\
\hline Cellulitis with deep myositis & $31(6.9)$ \\
\hline Pyomyositis & $31(6.9)$ \\
\hline Necrotizing fasciitis & $62(13.8)$ \\
\hline Traumatic infection & $12(2.7)$ \\
\hline Comorbid infection $^{\mathrm{b}}$ & $61(13.6)$ \\
\hline \multicolumn{2}{|l|}{ Depth of infection ${ }^{c}$} \\
\hline Subcutaneous & $192(42.8)$ \\
\hline Fascia & $66(14.7)$ \\
\hline Muscle & $107(23.8)$ \\
\hline Unclear & $84(18.7)$ \\
\hline \multicolumn{2}{|l|}{ Site of infection ${ }^{\mathrm{a}}$} \\
\hline Lower extremities & $211(44.1)$ \\
\hline Buttock & $80(16.7)$ \\
\hline Head and neck & $73(15 \cdot 3)$ \\
\hline Upper extremities & $74(15 \cdot 5)$ \\
\hline Trunk & $40(8.4)$ \\
\hline Admission via ER & $240(53.5)$ \\
\hline \multicolumn{2}{|l|}{ Clinical burden } \\
\hline Hospital length of stay, day & $16(8-28)$ \\
\hline ICU admission & $44(9.8)$ \\
\hline Recurrence after 3 months & $15(3 \cdot 3)$ \\
\hline In-hospital mortality & $19(4.2)$ \\
\hline
\end{tabular}

Values are presented as mean \pm SD or number (\%).

ER, emergency room; ICU, intensive care unit.

${ }^{\mathrm{a}}$ Multiple answers.

${ }^{b_{T}}$ The patients who had comorbid infection of more than 2 type of infection.

${ }^{\mathrm{c}}$ Deepest if multiple layers were involved.

\section{RESULTS}

\section{Characteristics of patients}

Patient characteristics are presented in Table 1. Of the 449 patients (mean age, 54 .6; 64.6\% male) who were admitted with a community-onset cSSSI, 415 (92.5\%) were $\leq 3$ in SEWS at the time of admission and 247 (55.0\%) had no comorbidities. The leading type of infection was major abscess ( $\mathrm{n}=233,51.9 \%$ ), followed by necrotizing cellulitis ( $n=144,31.1 \%)$. More than two infectious diagnoses were made in 61 (13.6\%) patients. The infections spread into subcutaneous tissues in 192 (42.8\%) patients, reached below fascia in 173 (38.5\%) patients and were most commonly found in the lower extremities $(\mathrm{n}=211$, 44.1\%). More than half of the cSSSI patients were admitted via the emergency room (ER) ( $n=240,53.5 \%)$.

\section{Clinical and economic burden}

The median duration of hospital stay for cSSSI treatment was 16 days (IQR, 8 to 28). Admission to the intensive care unit (ICU) was necessary for 44 (9.8\%) patients. Recurrence of disease within 3 months was observed in $15(3.3 \%)$ patients and $19(4.2 \%)$ cases of in-hospital mortality were recorded (Table 1 ).

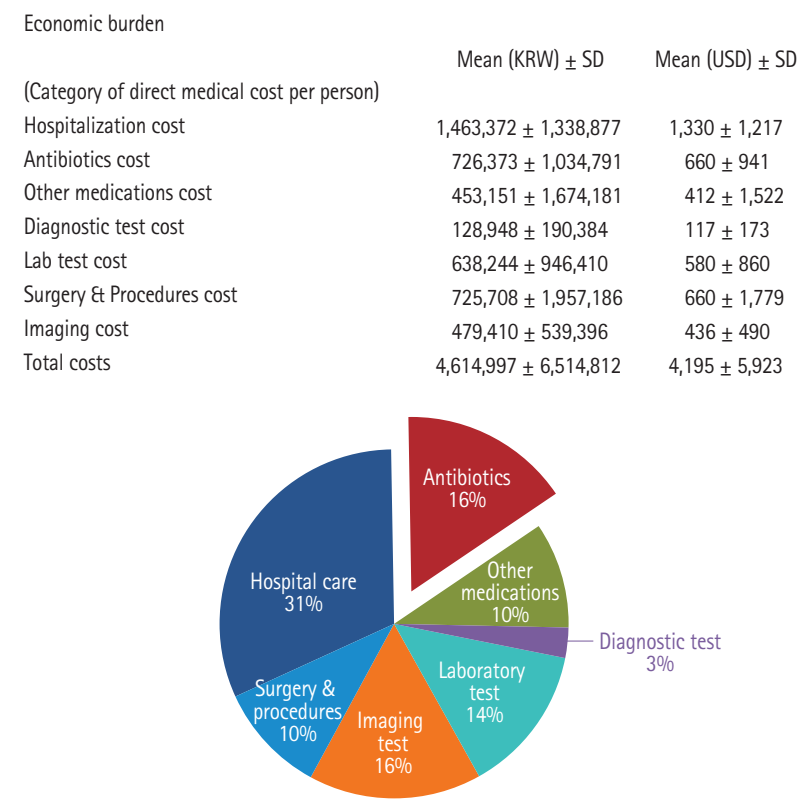

Figure 1. Economic burden of community-onset complicated skin and skin structure infections. KRW, Korean won; USD, United States dollar. 
Table 2. Distributions of pathogens of community-onset complicated skin and skin structure infections $(n=449)$

\begin{tabular}{|c|c|}
\hline Variable & No. (\%) \\
\hline No growth & $152(33.9)$ \\
\hline $\begin{array}{l}\text { Any pathogen identified by } \\
\text { microbiology test }\end{array}$ & $297(66.1)$ \\
\hline Monomicrobial & $260(57.9)$ \\
\hline Polymicrobial & $37(8.2)$ \\
\hline $\begin{array}{l}\geq 2 \text { Gram-positive pathogen } \\
\text { (no gram-negative pathogens) }\end{array}$ & $13(2.9)$ \\
\hline $\begin{array}{l}\geq 2 \text { Gram-negative pathogen } \\
\text { (no gram-positive pathogens) }\end{array}$ & $4(0.9)$ \\
\hline $\begin{array}{l}\text { Mixed (both gram-positive and } \\
\text {-negative pathogens) }\end{array}$ & $20(4 \cdot 5)$ \\
\hline \multicolumn{2}{|l|}{ Superinfection } \\
\hline No & $380(84.6)$ \\
\hline Yes & $69(15 \cdot 4)$ \\
\hline$\geq 2$ time & $19(4.2)$ \\
\hline \multicolumn{2}{|l|}{ Specimen type ${ }^{a}$} \\
\hline Blood & $26(8.6)$ \\
\hline Other & $277(91.4)$ \\
\hline \multicolumn{2}{|c|}{$\begin{array}{l}\text { Microbiology results: culture-confirmed } \\
\text { pathogens (episode case }=340)^{b}\end{array}$} \\
\hline Gram positive & $259(76.2)$ \\
\hline Staphylococcus aureus & $140(41.2)$ \\
\hline Group B Streptococcus & $31(9.1)$ \\
\hline
\end{tabular}

For cSSSI treatment, the mean total direct medical cost was $\$ 4,195 \pm 5,923$ per person. Hospitalization costs accounted for the maximum total direct medical cost, followed by the cost of antibiotics $(\$ 1,330 \pm 1,217$ and $\$ 660 \pm 941$, respectively). Costs for surgical procedures and imaging tests were also high with a mean of $\$ 660 \pm$ 1,779 and $\$ 436 \pm 490$, respectively (Fig. 1).

\section{Distribution of pathogens}

This study analyzed the tests of 449 patients whose specimens were obtained within 2 days after admission. As shown in Table 2, 297 (66.1\%) of 449 patients were identified by the microbiology test. Multiple pathogens were found in 37 (8.2\%) patients; 13 (2.9\%) had $\geq 2$ gram-positive pathogens, four $(0.9 \%) \mathrm{had} \geq 2$ gram-negative pathogens, and 20 (4.5\%) patients had both gram-positive and gram-negative pathogens (mixed pathogens). Superinfection was observed in 69 patients, and among those

\begin{tabular}{|cc}
\hline Variable & No. $(\%)$ \\
\hline Group A Streptococcus & $21(6.2)$ \\
\hline Enterococcus faecalis & $4(1.2)$ \\
\hline Others & $63(18.5)$ \\
\hline Gram negative & $81(23.8)$ \\
\hline Escherichia colic & $19(5.6)$ \\
Bacteroides fragilis & $3(0.9)$ \\
\hline Klebsiella species & $3(0.9)$ \\
\hline Proteus mirabilis & $10(2.9)$ \\
\hline Pseudomonas aeruginosa & $9(2.6)$ \\
Enterobacter species & $8(2.4)$ \\
Acinetobacter species & $6(1.8)$ \\
\hline Citrobacter species & $5(1.5)$ \\
\hline Others & $18(5.3)$ \\
\hline
\end{tabular}

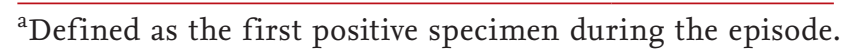
Not counted if no specimen were positive. "Other" specimens included tissue, wound drainage or aspirate, and other miscellaneous specimens taken from abscesses, pustules, etc.

${ }^{\mathrm{b}}$ Denominator for percent is the number of episodes with any positive pathogen (episode case $=340$ ). Pathogen results were not mutually-exclusive. The following organisms were considered contaminants and not included: coagulase negative Staphylococcus, Corynebacterium, Micrococcus spp., Bacillus spp., gram positive rods (episode case $=9$ ).

${ }^{\mathrm{c}}$ Extended spectrum $\beta$-lactamase producing bacteria was detected from Escherichia coli (1 case).

with superinfection 19 patients underwent two times of changes. Most specimens were taken from tissue, drainage or an aspirated wound, and other specimens were taken from abscesses and pustules, while only 26 (8.6\%) used blood cultures. Among culture confirmed pathogens (episode $=340$ ) which did not include coagulase negative Staphylococcus, Corynebacterium, Micrococcus spp., Bacillus spp. and gram-positive rods (episode = 9), most of the infections were caused by gram-positive bacteria (episode $=259,76.2 \%$ ) which were mainly $S$. aureus (episode $=140,41.2 \%$ ) and group B Streptococcus (episode = 31, 9.1\%). There were 81 (23.8\%) episodes of gram-negative bacterial infections.

\section{Comparison by initial therapy}

Initial therapy consisted of monotherapy and combined therapy. Monotherapy and combination therapy were determined according to the number of antibiotics in 
Table 3. Comparison of clinical characteristics and economic burden by initial therapy for community-onset complicated skin and skin structure infections

\begin{tabular}{|c|c|c|c|}
\hline Characteristic & Monotherapy $^{\mathrm{a}}(\mathrm{n}=202)$ & Combination therapy $^{\mathrm{b}}(\mathrm{n}=247)$ & $p$ value $^{c}$ \\
\hline Charlson comorbidity index & & & 0.719 \\
\hline $0-2$ & $179(88.6)$ & $215(87.0)$ & \\
\hline$\geq 3$ & $23(11.4)$ & $32(13.0)$ & \\
\hline Severity (SEWS) & & & 0.316 \\
\hline $0-3$ & $190(94.1)$ & $225(91.1)$ & \\
\hline$\geq 4$ & $12(5 \cdot 9)$ & $22(8.9)$ & \\
\hline Depth of infection & & & 0.217 \\
\hline Subcutaneous & $131(64.9)$ & $145(58.7)$ & \\
\hline below fascia & $71(35 \cdot 1)$ & $102(41.3)$ & \\
\hline \multicolumn{4}{|l|}{ Clinical burden } \\
\hline Hospital length of stay, day & $14(8-25)$ & $19(9-30)$ & $0.057^{\mathrm{d}}$ \\
\hline ICU admission & $14(6.9)$ & $30(12.1)$ & 0.091 \\
\hline Recurrence after 3 mon & $10(5.1)$ & $5(2.2)$ & 0.118 \\
\hline In-hospital mortality & $4(2.0)$ & $15(6.1)$ & 0.035 \\
\hline \multicolumn{4}{|l|}{ Economic burden ${ }^{\mathrm{e}}$} \\
\hline Total direct medical cost per person, \$ & $3,480 \pm 3,307$ & $4,781 \pm 7,361$ & 0.013 \\
\hline Antibiotics cost, $\$$ & $526 \pm 642$ & $770 \pm 1,117$ & 0.004 \\
\hline
\end{tabular}

Values are presented as number (\%), median (interquartile range), or mean $\pm \mathrm{SD}$.

SEWS, standardized early warning scoring; ICU, intensive care unit.

${ }^{a}$ Monotherapy and combination therapy were determined according to the number of antibiotics in treatment. In regardless of class counted in antibiotics, using only one antibiotic drug was defined as monotherapy whereas using multiple antibiotics was considered as combination therapy.

${ }^{\mathrm{b}}$ Using multiple antibiotics.

${ }^{c} p$ value by chi-square test.

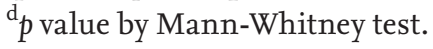

e $p$ value by $t$ test.

treatment. Regardless of the class counted in antibiotics, using only one antibiotic drug was defined as monotherapy whereas using multiple antibiotics was considered combination therapy. The proportion of $\mathrm{CCI} \geq 3$ in monotherapy and combined therapy were similar (11.4\% vs. $13.0 \%$ ). The proportion of SEWS $\geq 4$ and deep fascial space infections also were not significantly different (SEWS $\geq 4: 5.9 \%$ vs. 8.9\%; and deep fascial space infections: $35.1 \%$ vs. $41.3 \%$, respectively). The median length of hospital stay for cSSSI treatment with combined therapy (19 days) was longer than but not significantly different from monotherapy (14 days). Admissions to the ICU in patients treated with monotherapy (6.9\%) were lower than combined therapy (12.1\%), but a higher disease recurrence within 3 months was observed in patients treated with monotherapy (5.1\%) than combined therapy (2.2\%). However, these differences were not significant. The proportion of in-hospital mortality in patients treated with combined therapy (6.1\%) was significantly higher than monotherapy (2.0\%).

The total direct medical costs were compared according to the initial therapy for cSSSI treatment. The mean total cost for combined therapy was $\$ 4,781 \pm 7,361$ per person, which was higher than monotherapy $(\$ 3,480$ \pm 3,307 per person). The antibiotic costs for combined therapy were also higher than monotherapy $(\$ 770 \pm 1,117$ and $\$ 526 \pm 642$, respectively). Both the mean total cost per person and antibiotic cost were significantly higher in combined therapy than monotherapy (Table 3). 
Table 4. Factors related with mortality in community-onset complicated skin and skin structure infections

\begin{tabular}{|c|c|c|c|c|c|c|}
\hline \multirow{2}{*}{ Characteristic } & \multirow{2}{*}{$\begin{array}{c}\text { Death } \\
(\mathrm{n}=19)\end{array}$} & \multirow{2}{*}{$\begin{array}{l}\text { Survival }^{\mathrm{a}} \\
(\mathrm{n}=430)\end{array}$} & \multicolumn{2}{|c|}{ Univariate } & \multicolumn{2}{|c|}{ Multivariate } \\
\hline & & & OR (95\% CI) & $p$ value & OR (95\% CI) & $p$ value $^{\mathrm{b}}$ \\
\hline \multicolumn{7}{|l|}{ Sex } \\
\hline Female & $4(2.5)$ & $155(97 \cdot 5)$ & Ref & & & \\
\hline Male & $15(5.2)$ & $275(94.8)$ & $2.11(0.69-6.48)$ & 0.190 & $2.72(0.63-11.81)$ & 0.181 \\
\hline \multicolumn{7}{|l|}{ Age, yr } \\
\hline$<65$ & $11(3 \cdot 7)$ & $290(96.3)$ & Ref & & & \\
\hline$\geq 65$ & $8(5.4)$ & $140(94.6)$ & $1.51(0.59-3.83)$ & 0.389 & $3.38(1.00-11.48)$ & 0.051 \\
\hline \multicolumn{7}{|l|}{ Admission via ER } \\
\hline No & $2(1.0)$ & $207(99.0)$ & Ref & & & \\
\hline Yes & $17(7.1)$ & $223(92.9)$ & $7.89(1.80-34.57)$ & 0.006 & $2.53(0.48-13.31)$ & 0.274 \\
\hline \multicolumn{7}{|l|}{ Charlson comorbidity index } \\
\hline$<3$ & $10(2.5)$ & $284(97.5)$ & Ref & & & \\
\hline$\geq 3$ & $9(16.4)$ & $46(83.6)$ & $7.51(2.90-19.45)$ & $<0.001$ & $6.32(2.00-19.99)$ & 0.002 \\
\hline \multicolumn{7}{|l|}{ Severity (SEWS) } \\
\hline$<4$ & $9(2.2)$ & $406(97.8)$ & Ref & & & \\
\hline$\geq 4$ & $10(29.4)$ & $24(70.6)$ & $18.80(6.98-50.60)$ & $<0.001$ & $7.99(2.41-26.55)$ & 0.001 \\
\hline \multicolumn{7}{|l|}{ Depth of infection } \\
\hline Subcutaneous & $2(0.7)$ & $274(99 \cdot 3)$ & Ref & & & \\
\hline Below fascia & $17(9.8)$ & $156(90.2)$ & $14.93(3.40-65.47)$ & $<0.001$ & $8.95(1.78-45.06)$ & 0.008 \\
\hline \multicolumn{7}{|l|}{ Pathogen } \\
\hline Gram (+) only & $10(4 \cdot 5)$ & $211(95 \cdot 5)$ & $\operatorname{Ref}$ & & & \\
\hline Gram (-) only & $3(5 \cdot 4)$ & $53(94.6)$ & $0.99(0.27-3.61)$ & 0.991 & & \\
\hline Both Gram $(+)$ \& gram $(-)$ & $3(15 \cdot 0)$ & $17(85.0)$ & $3.58(0.93-13.79)$ & 0.063 & & \\
\hline \multicolumn{7}{|l|}{ Initial therapy } \\
\hline Monotherapy & $4(2.0)$ & $198(98.0)$ & Ref & & & \\
\hline Combined therapy & $15(6.1)$ & $232(93.9)$ & $3.20(1.05-9.80)$ & 0.042 & $3.66(1.00-13.33)$ & 0.050 \\
\hline \multicolumn{7}{|l|}{ Superinfection } \\
\hline No & $11(2.9)$ & $369(97.1)$ & Ref & & & \\
\hline Yes & $8(11.6)$ & $61(88.4)$ & $4.40(1.70-11.38)$ & 0.002 & $2.36(0.73-7.68)$ & 0.153 \\
\hline
\end{tabular}

Values are presented as number (\%).

OR, odds ratio; CI, confidence interval; ER, emergency room; SEWS, standardized early warning scoring.

${ }^{a}$ Values are number of patients and percentage in each group; $p$ value by logistic regression.

${ }^{b} p=0.942$ by Hosmer and Lemeshow test.

\section{Factors related to mortality in cSSSI}

In terms of mortality factors, admission via the ER (odds ratio [OR], 7.89; $p=0.006), \mathrm{CCI} \geq 3$ (OR, 7.51; $p<0.001$ ), SEWS $\geq 4$ (OR, 18.80; $p<0.001)$, deep fascial space infections (OR, 14.93; $p<0.001$ ), combination therapy as initial therapy (OR, 3.20; $p=0.042)$ and the presence of pathogen change (OR, 4.40; $p=0.002$ ) were significantly related to mortality as revealed by the univariate anal- ysis by logistic regression. In the multivariate analysis adjusted by age, sex, and the statistically significant factor noted in the univariate analysis, most factors were not significantly associated with mortality, except for comorbidity index, severity and depth of infection. In addition, combined initial therapy was marginally significant. These associations are presented in Table 4. 


\section{DISCUSSION}

In the present study, we identified epidemiological characteristics, clinical/economic burden and the factors associated with the burdens of community-onset cSSSIs patients in Korea. Of the detected cSSSIs, major abscess and cellulitis were predominant. More than one-third of the patients had deep fascial space infections. Approximately $40 \%$ of culture confirmed pathogens were $S$. aureus. These results are consistent with previous epidemiologic studies in Europe and the United States $[10,12]$.

The clinical burden of community-onset cSSSI observed in our study was notable. The median hospitalization period was $>2$ weeks and almost $10 \%$ of the patients were admitted to the ICU. In addition, 3.3\% of patients had a relapse within three months after treatment, and $4.2 \%$ of patients died while admitted to the hospital. A similar mortality rate is reported in a previously conducted European study [10]. The factors indicating more severe conditions at baseline such as higher CCI score ( $\geq 3$ ), higher SEWS $(\geq 4)$ and infection below fascia were associated with increased mortality risk.

In addition to the baseline severity of infection, a high economic burden of disease was seen. The total medical costs were $\$ 4,195$ per person. These costs for cSSSI treatment are much lower than those reported in the USD $\$ 48,033$ [11], especially when considering the public reimbursement system by the Korean government and economic gap between Korea and the United States. Over $50 \%$ of the total medical costs were comprised of costs for admission and treatment in this study. Nevertheless, total costs and mean cost of antibiotics for combined therapy were relatively higher than monotherapy. Since higher clinical and economic burdens of CSSSI were proven, it is essential to investigate the potential factors associated with severe disease burdens. Among many factors, the failure of initial antibiotic therapy was found to be significantly related to the unfavorable clinical and economic outcomes [13]. In this study, we categorized the initial therapy into two types, monotherapy versus combination therapy, and compared their clinical and economic outcomes. As a result, patients treated with combination therapy showed longer hospital stays and higher in-hospital mortality than those on monotherapy. Likewise, medical costs were higher in combi- nation therapy than monotherapy. However, direct association or causal relationship between two therapeutic types and disease outcomes might be clarified in further studies which should be well designed prospectively.

Several studies investigating epidemiological characteristics, clinical outcomes, and treatment cost of cSSSI patients have been conducted in foreign countries. To the best of our knowledge, this is the very first study that observed epidemiological characteristics, clinical and economic burden of community-onset cSSSI patients, reflecting real world clinical practice in Korea. Furthermore, since this study was a nationwide study conducted in multiple centers across Korea, its outcomes are representative of the population and are applicable to the general population. It follows that the results of this study are expected to improve awareness of the disease burden of community-onset cSSSIs in Korean patients, and will encourage clinicians to select appropriate monotherapy as the initial treatment option.

This study has several limitations, some of which stem from its retrospective design. This study excluded a large number of patients with cSSSIs who did not complete treatment in the hospital; this was important since this study focused on the disease burden from diagnosis to treatment completion. In addition, this study did not consider the events that occurred outside of the hospital, so the mortality rate should be interpreted carefully. Moreover, it should be acknowledged that due to the nature of cost data in non-normal distribution, the data on medical cost was skewed to one side, which resulted in the large standardized deviation. So, the direct medical costs should be cautiously evaluated. Finally, only direct medical costs were considered, and thus, the actual economic burden due to cSSSIs in Korean patients is higher when indirect costs are considered. Further study of these medical expenses could provide a holistic understanding of cSSSI management from the Korean context.

\section{KEY MESSAGE}

1. The epidemiological characteristics of Korean community-onset complicated skin and skin structure infection (cSSSI) patients were similar to patients with cSSSI in other countries. Korean patients with community-onset cSSSI suffer 
from substantial clinical and economic burden; they were admitted to the hospital for approximately 16 days, the infection recurred in 3.3\% of the patients within 3 months post treatment, $4.2 \%$ of the patients died, and overall $\$ 4,195$ was spent per capita for cSSSI treatment.

2. The mortality rate increased in patients with more severe baseline characteristics. This highlights the importance of reducing patients' burden of disease through appropriate and simple initial treatment.

\section{Conflict of interest}

This study was sponsored by Pfizer Pharmaceuticals Korea Ltd.

\section{Acknowledgments}

The following investigators also participated in the study: Yong Kyun Cho, Department of Internal Medicine, Gachon University Gil Hospital; Jacob Lee, Department of Internal Medicine, Kangnam Sacred Heart Hospital; Heung Jeong Woo, Department of Internal Medicine, Hangang Sacred Heart Hospital; Seong Yeol Ryu, Department of Internal Medicine, Keimyung University Dongsan Medical Center; Chi Sook Moon, Department of Internal Medicine, Inje University Pusan Paik Hospital; Sang Hoon Han, Department of Internal Medicine, Severance Hospital, Yonsei University Health System; Young Goo Song, Department of Internal Medicine, Kangnam Severance Hospital, Yonsei University College of Medicine; You Mi Jo, Department of Internal Medicine, Korea University Guro Hospital; Shin Woo Kim, Department of Medicine, Kyungpook National University Hospital; Hyuck Lee, Department of Internal Medicine, Dong-A University Hospital; In Gyu Bae, Infection Control Office, Gyeongsang National University Hospital; Jian Hur, Department of Medicine, Yeungnam University Medical Center; Hee Jung Choi, Department of Medicine, Ewha Womans University Mokdong Hospital; Hee Jin Cheong, Department of Internal Medicine, Korea University Guro Hospital.

\section{REFERENCES}

1. Stevens DL, Bisno AL, Chambers HF, et al. Practice guidelines for the diagnosis and management of skin and soft tissue infections: 2014 update by the Infectious Diseases Society of America. Clin Infect Dis 2014;59:e10-e52.

2. Center for Drug Evaluation and Research, Food and Drug Administration. Guidance for Industry: Uncomplicated and Complicated Skin and Skin Structure InfectionsDeveloping Antimicrobial Drugs for Treatment. Washington, DC: Food and Drug Administration, US Department of Health and Human Services, 1998.

3. May AK, Stafford RE, Bulger EM, et al. Treatment of complicated skin and soft tissue infections. Surg Infect (Larchmt) 2009;10:467-499.

4. Nichols RL. Optimal treatment of complicated skin and skin structure infections. J Antimicrob Chemother 1999;44:19-23.

5. DiNubile MJ, Lipsky BA. Complicated infections of skin and skin structures: when the infection is more than skin deep. J Antimicrob Chemother 2004;53:ii37-ii5o.

6. Doern GV, Jones RN, Pfaller MA, Kugler KC, Beach ML. Bacterial pathogens isolated from patients with skin and soft tissue infections: frequency of occurrence and antimicrobial susceptibility patterns from the SENTRY Antimicrobial Surveillance Program (United States and Canada, 1997). SENTRY Study Group (North America). Diagn Microbiol Infect Dis 1999;34:65-72.

7. Lee SY, Kuti JL, Nicolau DP. Antimicrobial management of complicated skin and skin structure infections in the era of emerging resistance. Surg Infect (Larchmt) 2005;6:283-295.

8. Hook EW 3rd, Hooton TM, Horton CA, Coyle MB, Ramsey PG, Turck M. Microbiologic evaluation of cutaneous cellulitis in adults. Arch Intern Med 1986;146:295-297.

9. McHenry CR, Piotrowski JJ, Petrinic D, Malangoni MA. Determinants of mortality for necrotizing soft-tissue infections. Ann Surg 1995;221:558-563.

10. Garau J, Ostermann H, Medina J, et al. Current management of patients hospitalized with complicated skin and soft tissue infections across Europe (2010-2011): assessment of clinical practice patterns and real-life effectiveness of antibiotics from the REACH study. Clin Microbiol Infect 2013;19:E377-E385.

11. Itani KM, Merchant S, Lin SJ, Akhras K, Alandete JC, Hatoum HT. Outcomes and management costs in patients 
hospitalized for skin and skin-structure infections. Am J Infect Control 2011;39:42-49.

12. Zilberberg MD, Shorr AF, Micek ST, et al. Epidemiology and outcomes of hospitalizations with complicated skin and skin-structure infections: implications of healthcare-associated infection risk factors. Infect Control Hosp
Epidemiol 2009;30:1203-1210.

13. Edelsberg J, Berger A, Weber DJ, Mallick R, Kuznik A, Oster G. Clinical and economic consequences of failure of initial antibiotic therapy for hospitalized patients with complicated skin and skin-structure infections. Infect Control Hosp Epidemiol 2008;29:160-169. 